\title{
Resilience Enhancement of Distribution Grids Against Extreme Weather Events
}

\author{
Shanshan Ma, Student Member, IEEE, Liu Su, Zhaoyu Wang, Member, IEEE, Feng Qiu, Member, IEEE, Ge Guo
}

\begin{abstract}
This paper proposes a resilience-oriented design (ROD) technique to protect distribution grids against high-impact but low-probability extreme weather events. The problem is formulated as a two-stage stochastic mixed integer problem. The first stage is to make ROD decisions, i.e., hardening existing distribution lines and deploying ROD resources such as backup distributed generators (DGs) and automatic switches. The second stage evaluates the system operation cost during a realized extreme weather event and repair cost after the event. A novel modeling strategy is proposed to deal with the decision-dependent uncertainty of distribution line damage status which is affected by the first-stage hardening decisions. As both stages have binary variables, a modified and computationally efficient progressive hedging algorithm with scenario bundling is introduced. The algorithm performance is evaluated by calculating lower bounds of solutions. The proposed model and algorithms are demonstrated on 34-bus and 123-bus test feeders.
\end{abstract}

Index Terms-Distribution systems, decision-dependent uncertainty, failure probability, progressive hedging, resilience-oriented design, scenario bundling, stochastic programming

\section{NOMENCLATURE}

\section{Sets and Indices}

$\Omega_{N} \quad$ Set of buses indices $i$

$\Omega_{B} \quad$ Set of lines indices $(i, j)$

$\Omega_{D G} \subset \Omega_{N}$ Set of buses with DGs

$\Omega_{S G} \subset \Omega_{N}$ Set of substation buses

$\Omega_{S W} \subseteq \Omega_{B} \quad$ Set of lines with switches

$\Omega_{L} \subseteq \Omega_{N} \quad$ Set of buses with loads

$\mathcal{T} \quad$ Set of durations of extreme weather events indices

$$
\mathcal{S} \quad \text { Set of scenarios indices } s
$$

\section{Parameters}

$c_{i j}^{c}$

$c_{i j}^{h}$

$c_{i}^{g}$

$c_{i}^{L}$

$c^{R_{0}}$

$M$

$N_{G}$

$P_{i, t}^{L, s}$

Annual capital cost $(\$)$ for adding an automatic tie switch at line $(i, j)$

Annual capital cost $(\$)$ for hardening line $(i, j)$

Annual capital cost $(\$)$ for deploying a back-up DG at bus $i$

Penalty cost $(\$)$ for shedding $1 \mathrm{kWh}$ load at bus $i$

\section{Base repair cost $(\$)$}

Sufficiently large positive number

The total number of newly installed DGs

Active load $(\mathrm{kW})$ at bus $i$ in time period $t$ at scenario $s$

S. Ma and Z. Wang are with the Department of Electrical and Computer Engineering, Iowa State University, Ames, IA, 50014. (Email:sma@iastate.edu,wzy@iastate.edu).

L. Su is with the Department of Industrial and Management Systems Engineering, University of South Florida, Tampa, FL 33620. (Email: liusu@mail.usf.edu).

F. Qiu is with the Energy Systems Division, Argonne National Laboratory, Lemont, IL 60439 USA (Email: fqiu@anl.gov).

G. Guo is with the Department of Industrial and Manufacturing Systems Engineering, Iowa State University, Ames, IA 50014. (Email:geguo@iastate.edu).
$Q_{i, t}^{L, s}$

$P_{i}^{g, \max }$

$Q_{i}^{g, \max }$

$V_{i}^{\max }$
$V_{i}^{\min }$

$P_{i}^{\max }$

$P_{i j}^{\max }$

$Q_{i j}^{\max }$

$R_{i j}$

$T_{i j}^{R, s}$

$V_{0}^{i j}$

$X_{i j}$

$x_{i j}^{c_{0}}$

$\omega_{H}$

$\zeta_{i j, t}^{0}(s)$

$\zeta_{i j, t}^{1}(s)$

$t_{i j}^{d, 0}(s)$

$t_{i j}^{d, 1}(s)$

$\Delta t$

Variables

$c_{i j}^{R, s}$

$\mathcal{C}_{2}^{I}\left(x^{g}\right)$

$\mathcal{C}_{3}^{I}\left(x^{c}\right)$

$P_{i j, t}^{s}$

$Q_{i j, t}^{s}$

$P_{i, t}^{g, s}$

$Q_{i, t}^{g, s}$

$x_{i j}^{c_{1}}$

$x_{i j}^{c}$

$x_{i j}^{h}$

$x_{i}^{g}$
Reactive load (kVar) at bus $i$ in time period $t$ at scenario $s$

Maximum active power limit $(\mathrm{kW})$ of generation at bus $i$

Maximum reactive power limit (kVar) of generation at bus $i$

Maximum voltage magnitude (pu) at bus $i$

Minimum voltage magnitude (pu) at bus $i$

Maximum active power limit $(\mathrm{kW})$ for line $(i, j)$

Maximum reactive power limit (kW) for line $(i, j)$

Resistance (pu) for line $(i, j)$

Repair time (hour) for line $(i, j)$ at scenario $s$

Reference voltage magnitude

Reactance (pu) for line $(i, j)$

Binary parameter indicating whether line $(i, j)$ has an exist switch (1) or not (0)

Average occurrences of extreme weather events in a year

Parameter indicating the damage status of line $(i, j)$ if it is not hardened, damaged (1) or functional (0) at time $t$ in scenario $s$

Parameter indicating the damage status of line $(i, j)$ if it is hardened, damaged (1) or functional (0) at time $t$ in scenario $s$

The first time that $\zeta_{i j, t}^{0}(s)=1$ occurs in scenario

The first time that $\zeta_{i j, t}^{1}(s)=1$ occurs in scenario

Time-period length

Repair cost $(\$)$ for line $(i, j)$ in scenario $s$

Total annual capital cost $(\$)$ of line hardening

Total annual capital cost (\$) of installing back-up DGs

Total annual capital cost $(\$)$ of adding new automatic tie switches

Active power flow $(\mathrm{kW})$ of line $(i, j)$ at time $t$ in scenario $s$

Reactive power flow (kVar) of line $(i, j)$ at time $t$ in scenario $s$

Active power output $(\mathrm{kW})$ of generation at bus $i$ at time $t$ in scenario $s$

Reactive power output (kVar) of generation at bus $i$ at time $t$ in scenario $s$

Binary variable indicating whether new line switch is added (1) or not (0) at line $(i, j)$

Binary variable indicating whether line $(i, j)$ has switch (1) or not (0)

Binary variable indicating whether line $(i, j)$ is hardened (1) or not (0)

Binary variable indicating whether a new back-up 


\begin{tabular}{|c|c|}
\hline$y_{i j, t}^{c, s}$ & $\begin{array}{l}\text { DG is placed at bus } i(1) \text { or not }(0) \\
\text { Binary variable indicating whether the line switch } \\
\text { at line }(i, j) \text { is closed }(1) \text { or not }(0) \text { at time } t \text { in } \\
\text { scenario } s\end{array}$ \\
\hline$\vartheta_{m n, t}^{s}$ & $\begin{array}{l}\text { Binary variable equals } 1 \text { if } m \text { is the parent bus of } \\
n \text { and } 0 \text { otherwise at time } t \text { in scenario } s\end{array}$ \\
\hline$u_{i j, t}^{s}$ & $\begin{array}{l}\text { Binary variable indicating whether line status is } \\
\text { damaged (1) or not }(0) \text { at time } t \text { in scenario } s\end{array}$ \\
\hline$\gamma_{i, t}^{s}$ & $\begin{array}{l}\text { Load shedding percentage of load } i \text { at time } t \text { in } \\
\text { scenario } s\end{array}$ \\
\hline$V_{i, t}^{s}$ & $\begin{array}{l}\text { Voltage magnitude of bus } i \text { at time } t \text { in scenario } \\
s\end{array}$ \\
\hline
\end{tabular}

\section{INTRODUCTION}

$\mathbf{P}$ RESENTLY, most distribution systems are designed and maintained for normal weather conditions, which cannot withstand extensive damages caused by extreme weather events [1]. For example, Hurricane Wilma in 2005 damaged more than ten-thousand distribution poles in Florida [1]. Hurricane Sandy in 2012 devastated the overhead distribution system in New York, which resulted in a loss of nearly 1000 utility poles and more than 900 transformers as well as $8,371,242$ customer outages [2]. In addition, U.S. power grids are now old and outdated, making them more vulnerable to such events [3]. One way to address this issue is to design resilient distribution systems that can withstand extreme weather events.

A distribution system is considered to be resilient if it is able to anticipate, absorb, adapt to, and/or rapidly recover from a disruptive event [4]. The resilience enhancement goals can be fulfilled through upgrading and operating measures [5]. Our paper focuses on exploring the impacts of upgrading grids on system resilience. Resilience-oriented design (ROD) is defined as topological and structural upgrades to make power system less susceptible to extreme weather events [5]. In general, hardening existing distribution lines, installation of DGs and adding line switches are effective ROD strategies that are commonly used by utilities. Hardening distribution lines with stronger materials can make them more robust to extreme weather events. Backup DGs can provide on-site power for critical facilities and load centers, and contribute to energizing microgrids to restore load after an extreme weather event $[6]-[11]$. Installing automatic switches enables network reconfiguration that can re-route power to on-outage portions of distribution networks, shorten the restoration time and enhance the restoration capability [12].

There are a few studies on ROD of distribution systems to protect against extreme weather events, which in general apply two types of modeling techniques: robust and stochastic modeling. Yuan et al. [13] proposed a new robust optimization model to solve the resilient distribution network planning problem under the worst scenario of extreme weather events. However, their study used a polyhedral set to represent damage uncertainty, where grid fragility models for specific extreme weather events were ignored in calculating uncertainty budget. Moreover, only one hardening measure, i.e., line hardening, was taken into consideration. In addition, it was assumed that hardened lines would not be damaged in future events, which is impractical. In [14], the authors proposed a power distribution system hardening framework using a tri-level robust optimization. Ref. [14] considered three hardening strategies: upgrading distribution poles, vegetation managements, and the combination of the two. A greedy algorithm was proposed to deal with the coupling issue of the hardening decisions in the first level and the damage uncertainty in the second level. However, some important ROD measures were missed, such as the installation of DGs and automatic tie switches.

Another approach to manage uncertainty in ROD problems is stochastic programming. Stochastic programming is an extension of standard deterministic mathematical programming, in which the space of possible outcomes (e.g. line failure uncertainty) is represented by a probability-weighted scenario tree. Yamangil et al. [15] proposed a scenario-based variable neighborhood decomposition search algorithm to design resilient electrical distribution grids. This work improved previous studies by assuming that the hardened lines could be damaged at the rate of $\frac{1}{10}$ of unhardened counterparts. However, the interrelation between the first-stage line hardening decisions, and the uncertain line damage status, was not taken into account. The decision-dependent uncertainty is inherent in infrastructure resilience enhancement. This is because the resilience enhancement can only change the failure probability of system components, but cannot reduce it to zero. To manage the risk in a more realistic manner, it is necessary to model the failure of system components as a decision-dependent uncertainty. Arab et al. [16] proposed a proactive resource allocation model for repair and restoration of potential damages to power system components located on the traveling paths of upcoming hurricanes. They used Bender's decomposition to solve the two-stage stochastic integer program. But the quality of solutions were not evaluated. Moreover, Bender's decomposition is efficient in solving stochastic programs with linear programming problems but not for mixed-integer programs in the second stage.

In this paper, we formulate the ROD problem using a twostage stochastic mixed-integer programming (SMIP) model. In the first stage, the system planner makes ROD decisions: hardening existing distribution lines and deploying ROD resources such as back-up distributed generators (DGs) and automatic tie switches. The second stage evaluates the system operation cost and repair cost under an extreme weather event. The enhancement of resilience under extreme weather events can be evaluated by the reduction in load shedding penalty cost and repair cost. There are two key challenges: $(i)$ the decisiondependent uncertainty, i.e., the first-stage hardening decisions will affect the resolution of line damage status uncertainty, is inherent in infrastructure resilience enhancement and difficult to model; (ii) the existence of integer constraints on the ROD decision making in the first stage and recourse decisions (operation statuses of tie switches and DGs, and damage status of distribution lines) in the second stage results in the nonconvexity of stochastic ROD models and considerable computation difficulties. This paper resolves the two challenges by providing the following key contributions:

(1) An innovative modeling technique is proposed to capture the decision-dependent uncertainty in ROD modeling. The uncertain line damage status is decoupled by two independent parameters, which can be generated in advance to represent the line damage status with and without hardening. A constraint is formulated to pick the two independent variables to represent the realized line damage status according to the hardening decisions. This method avoids a time-consuming and computationally intensive procedure of generating a large dictionary of scenario sets for all possible values of hardening decisions. Moreover, the inter-temporal correlation imposed by the repair process can be captured by sampling the two 
independent variables.

(2) Efficient algorithms based on the modified progressive hedging $(\mathrm{PH})$ are introduced to cope with the large-scale twostage SMIP ROD problems. The $\mathrm{PH}$ algorithm can reduce the computational difficulty by decomposing the problem into scenario-based subproblems and solving subproblems in parallel. We apply a scenario bundling technique to significantly improve the quality of PH algorithm's lower bounds. A multiple replication procedure (MRP) is applied to test the stability and quality of candidate solutions with limited scenarios.

The rest of the paper is organized as follows. Section II proposes mathematical formulations. Section III provides two solution algorithms. Numerical results are presented in Section IV. Section V concludes the study.

\section{Mathematical Formulation}

We propose a two-stage stochastic mixed-integer programming framework for ROD under uncertainty. The first stage is to make ROD decisions (hardening existing components and allocating ROD resources such as DGs and switches), and the second stage is to evaluate operation costs in terms of load shedding and damage repair in realized extreme weather events, e.g., hurricanes, ice storms, etc. In this section, we will first present the overall formulation for the two-stage stochastic program of ROD, then describe in detail the model for uncertainties, and the model for the recourse behavior of the distribution system in an extreme weather event.

\section{A. Optimization Model}

$$
\begin{gathered}
\min \mathcal{C}_{1}^{I}\left(x^{h}\right)+\mathcal{C}_{2}^{I}\left(x^{g}\right)+\mathcal{C}_{3}^{I}\left(x^{c_{1}}\right)+\omega_{H} \mathbb{E}_{s} \phi(s) \\
\text { s.t. } \quad \sum_{i \in \Omega_{D G}} x_{i}^{g} \leqslant N_{G} \\
x_{i j}^{c_{0}}+x_{i j}^{c_{1}}=x_{i j}^{c}, \forall(i, j) \in \Omega_{S W} \\
x_{i}^{g}, x_{i j}^{h}, x_{i j}^{c}, x_{m n}^{c 1} \in\{0,1\}, \forall i \in \Omega_{D G},(i, j) \in \Omega_{B},(m, n) \in \Omega_{S W}
\end{gathered}
$$

where

$$
\begin{aligned}
\mathcal{C}_{1}^{I}\left(x^{h}\right) & =\sum_{(i, j) \in \Omega_{B}} c_{i j}^{h} x_{i j}^{h} \\
\mathcal{C}_{2}^{I}\left(x^{g}\right) & =\sum_{i \in \Omega_{D G}} c_{i}^{g} x_{i}^{g} \\
\mathcal{C}_{3}^{I}\left(x^{c_{1}}\right) & =\sum_{(i, j) \in \Omega_{S W}} c_{i j}^{c} x_{i j}^{c_{1}} \\
\mathbb{E}_{s} \phi(s) & =\sum_{s \in \mathcal{S}} p_{r}(s) \phi(s)
\end{aligned}
$$

with

$$
\begin{gathered}
\phi(s)=\min \sum_{t \in \mathcal{T}} \sum_{i \in \Omega_{L}} c_{i}^{L} \gamma_{i, t}^{s} P_{i, t}^{L, s} \Delta t+\sum_{(i, j) \in \Omega_{B}} c_{i j}^{R, s} \\
\text { s.t. } y_{i j, t}^{c, s} \leqslant x_{i j}^{c}, \forall(i, j) \in \Omega_{S W}, t \in \mathcal{T} \\
u_{i j, t}^{s}=\left(1-x_{i j}^{h}\right) \zeta_{i j, t}^{0, s}+x_{i j}^{h} \zeta_{i j, t}^{1, s}, \forall(i, j) \in \Omega_{B}, t \in \mathcal{T} \\
c_{i j}^{R, s}=c^{R_{0}} \cdot \sum_{t \in \mathcal{T}} u_{i j, t}^{s}, \forall(i, j) \in \Omega_{B}
\end{gathered}
$$

$$
\begin{aligned}
& \sum_{\left\{j \mid(i, j) \in \Omega_{B}\right\}} P_{i j, t}^{s}-\sum_{\left\{j \mid(i, j) \in \Omega_{B}\right\}} P_{j i, t}^{s}=P_{i, t}^{g, s}-\left(1-\gamma_{i, t}^{s}\right) P_{i, t}^{L, s}, \\
& \forall i \in \Omega_{N}, t \in \mathcal{T} \\
& \sum_{\left\{j \mid(i, j) \in \Omega_{B}\right\}} Q_{i j, t}^{s}-\sum_{\left\{j \mid(i, j) \in \Omega_{B}\right\}} Q_{j i, t}^{s}=Q_{i, t}^{g, s}-\left(1-\gamma_{i, t}^{s}\right) Q_{i, t}^{L, s}, \\
& \forall i \in \Omega_{N}, t \in \mathcal{T} \\
& y_{i j, t}^{c, s}+u_{i j, t}^{s} \leqslant 1, \forall(i, j) \in \Omega_{S W}, t \in \mathcal{T} \\
& -y_{i j, t}^{c, s} P_{i j}^{\max }-\left(1-x_{i j}^{c}\right) M \leqslant P_{i j, t}^{s} \leqslant y_{i j, t}^{c, s} P_{i j}^{\max } \\
& +\left(1-x_{i j}^{c}\right) M, \forall(i, j) \in \Omega_{S W}, t \in \mathcal{T} \\
& -y_{i j, t}^{c, s} Q_{i j}^{\max }-\left(1-x_{i j}^{c}\right) M \leqslant Q_{i j, t}^{s} \leqslant y_{i j, t}^{c, s} Q_{i j}^{\max } \\
& +\left(1-x_{i j}^{c}\right) M, \forall(i, j) \in \Omega_{S W}, t \in \mathcal{T} \\
& -\left(1-u_{i j, t}^{c, s}\right) P_{i j}^{\max }-x_{i j}^{c} M \leqslant P_{i j, t}^{s} \leqslant\left(1-u_{i j, t}^{c, s}\right) P_{i j}^{\max } \\
& +x_{i j}^{c} M, \forall(i, j) \in \Omega_{B}, t \in \mathcal{T} \\
& -\left(1-u_{i j, t}^{c, s}\right) Q_{i j}^{\max }-x_{i j}^{c} M \leqslant Q_{i j, t}^{s} \leqslant\left(1-u_{i j, t}^{c, s}\right) Q_{i j}^{\max } \\
& +x_{i j}^{c} M, \forall(i, j) \in \Omega_{B}, t \in \mathcal{T} \\
& V_{i, t}^{s}-\frac{R_{i j} P_{i j, t}^{s}+X_{i j} Q_{i j, t}^{s}}{V_{0}}-\left(1-y_{i j, t}^{c, s}+1-x_{i j}^{c}\right) M \leqslant V_{j, t}^{s} \\
& \leqslant V_{i, t}^{s}-\frac{R_{i j} P_{i j, t}^{s}+X_{i j} Q_{i j, t}^{s}}{V_{0}}+\left(1-y_{i j, t}^{c, s}+1-x_{i j}^{c}\right) M, \\
& \forall(i, j) \in \Omega_{S W}, t \in \mathcal{T} \\
& V_{i, t}^{s}-\frac{R_{i j} P_{i j, t}^{s}+X_{i j} Q_{i j, t}^{s}}{V_{0}}-\left(u_{i j, t}^{s}+x_{i j}^{c}\right) M \leqslant V_{j, t}^{s} \\
& \leqslant V_{i, t}^{s}-\frac{R_{i j} P_{i j, t}^{s}+X_{i j} Q_{i j, t}^{s}}{V_{0}}+\left(u_{i j, t}^{s}+x_{i j}^{c}\right) M, \\
& \forall(i, j) \in \Omega_{B}, t \in \mathcal{T} \\
& \vartheta_{m n, t}^{s}+\vartheta_{n m, t}^{s}-x_{i j}^{c} M \leqslant 1-u_{i j, t}^{s} \leqslant \vartheta_{m n, t}^{s}+\vartheta_{n m, t}^{s} \\
& +x_{i j}^{c} M, \forall(i, j) \in \Omega_{B}, t \in \mathcal{T} \\
& \vartheta_{m n, t}^{s}+\vartheta_{n m, t}^{s}-\left(1-x_{i j}^{c}\right) M \leqslant y_{i j, t}^{c, s} \leqslant \vartheta_{m n, t}^{s}+\vartheta_{n m, t}^{s} \\
& +\left(1-x_{i j}^{c}\right) M, \forall(i, j) \in \Omega_{S W}, t \in \mathcal{T} \\
& \vartheta_{m n, t}^{s}=0, \forall(m, n) \in \Omega_{S G}, t \in \mathcal{T} \\
& \sum_{n \in N(m)} \vartheta_{m n, t}^{s} \leqslant 1, \forall m \in \Omega_{N}, t \in \mathcal{T} \\
& \vartheta_{m n, t}^{s} \in\{0,1\}, \forall(m, n) \in \Omega_{B}, t \in \mathcal{T} \\
& 0 \leqslant P_{i, t}^{g, s} \leqslant x_{i}^{g} P_{i}^{g, \max }, \forall i \in \Omega_{D G}, t \in \mathcal{T} \\
& 0 \leqslant Q_{i, t}^{g, s} \leqslant x_{i}^{g} Q_{i}^{g, \max }, \forall i \in \Omega_{D G}, t \in \mathcal{T} \\
& P_{i, t}^{g, s} \geqslant 0, \forall i \in \Omega_{S G}, t \in \mathcal{T} \\
& Q_{i, t}^{g, s} \geqslant 0, \forall i \in \Omega_{S G}, t \in \mathcal{T} \\
& V_{i}^{\min } \leqslant V_{i, t}^{s} \leqslant V_{i}^{\max }, \forall i \in \Omega_{N}, t \in \mathcal{T} \\
& 0 \leqslant \gamma_{i, t}^{s} \leqslant 1, \forall i \in \Omega_{L}, t \in \mathcal{T} \\
& y_{i j, t}^{c, s}, u_{i j, t}^{s} \in\{0,1\}, \forall(i, j) \in \Omega_{B}, t \in \mathcal{T}
\end{aligned}
$$

In this two-stage stochastic programming model, the objective is to minimize ROD investment cost and the product of the annual occurrence of extreme weather events and the 
expected costs of the projected load shedding and damage repair with respect to the probability distribution of scenarios, as shown in equation (1). The investment cost of hardening lines, placing DGs and adding line switches is given by (5)(7), respectively, which includes purchasing and installation cost. Constraint (2) restricts the number of DGs that can be installed. $x_{i j}^{c}$ in constraint (3) represents whether line $(i, j)$ has a switch or not, which will be used in the second-stage problem. $x_{i j}^{c_{1}}$ represents a first-stage decision, i.e., whether to add a new switch at line $(i, j)$. If there is an existing switch on line $(i, j), x_{i j}^{c_{0}}=1$, then it is unnecessary to install a new switch, which imposes $x_{i j}^{c_{1}}=0$. If $x_{i j}^{c_{0}}=0$, it indicates that whether line $(i, j)$ has a new switch or not depends on the first-stage decision $x_{i j}^{c_{1}}$. Here $x_{i j}^{c_{0}}$ is assumed to be a known parameter.

The expectation of load shedding penalty cost obtained by solving the second-stage problem is described in equation (8), where $p_{r}(s)$ is defined as the probability of $s$ being realized (approximated by the frequency in $\mathcal{S}$ ), and $\mathbb{E}_{s}$ denotes the mathematical expectation with respect to $s$. For a fixed value of first-stage decision variables $\boldsymbol{x}=\left[\boldsymbol{x}^{h}, \boldsymbol{x}^{c}, \boldsymbol{x}^{g}\right]$ and a particular scenario $s$, a second-stage problem can be described by equations (9)-(33). Equation (9) represents the objective function of the second-stage problem, i.e., minimizing the total load shedding cost during an extreme weather event, and the damage repair cost after the event. Constraint $(10)$ indicates that if a switch has been added in the first stage, the switch can be used for reconfiguration in the second stage under a specific scenario, which connects first-stage decisions with second-stage variables. Constraints (11)-(33) will be discussed in Sections $\amalg I-B$ and $\amalg-C$.

\section{B. Uncertainty Modeling and Scenario Generation}

This paper considers four random variables for the stochastic scenario $s$ : the load multiplier, the wind speed, the line repair time, and the line damage status.

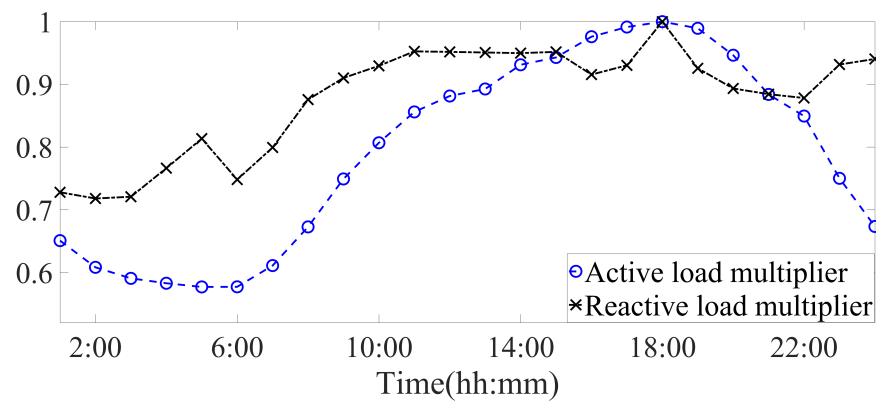

Fig. 1. Multipliers of load profiles at the substation (root node), with the peak values as the bases.

1) Uncertainty of load: $\tau(s)$ describes the load level uncertainty for a stochastic scenario. Fig. 11 shows the multipliers of active and reactive load profiles at a substation on a typical day in summer [17], [18], denoted by $M^{P}(t)$ and $M^{Q}(t)$. The active and reactive load are normalized with respect to their peak values respectively. We make an assumption of homogeneous load pattern: all individual node loads share the same normalized active and reactive load profiles [19]. The base values of each load, denoted by $P_{i}$ and $Q_{i}$, are given by the test system.

$$
\begin{aligned}
& P_{i, t}^{L, s}=\tau_{i}(s) \cdot M^{P}(t) \cdot P_{i}, \forall i \in \Omega_{L}, t \in \mathcal{T} \\
& Q_{i, t}^{L, s}=\tau_{i}(s) \cdot M^{Q}(t) \cdot Q_{i}, \forall i \in \Omega_{L}, t \in \mathcal{T} .
\end{aligned}
$$

where $\tau_{i}(s)$ is assumed to follow a normal distribution [20].

2) Uncertainty of wind-induced extreme events: In this paper, we consider wind-induced extreme weather events (e.g. hurricanes), since they pose the top threat to distribution systems. $v(s)$ represents the wind speed. Here we use hurricanes for illustration. During a hurricane, the wind speed at a distribution line can be represented by a function of the distance from the distribution line to the hurricane eye as shown in Fig. 2] [21]:

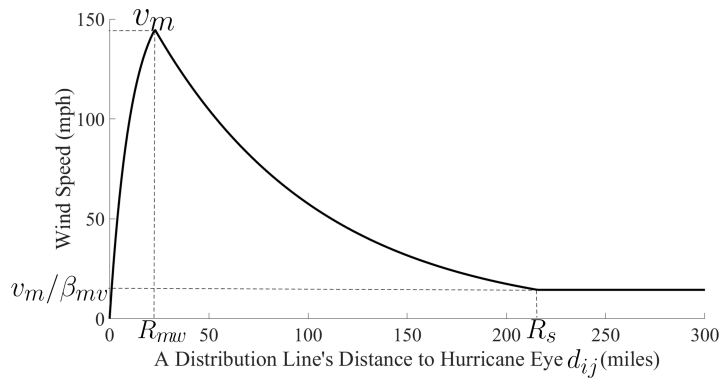

Fig. 2. The simulated wind speeds at different distribution lines during a hurricane

$v_{i j}(s)= \begin{cases}K_{v} v_{m}\left(1-\exp \left[-\frac{1}{R_{m w}} \ln \left(\frac{K_{v}}{K_{v}-1}\right) d_{i j}\right]\right) & 0 \leqslant d_{i j}<R_{m v} \\ v_{m} \exp \left[-\left(\frac{\ln \beta_{m v}}{R_{s}-R_{m w}}\right)\left(d_{i j}-R_{m v}\right)\right. & R_{m v} \leqslant d_{i j} \leqslant R_{s} \\ 0 & d_{i j}>R_{s}\end{cases}$

where parameter $K_{v}=1.14 ; v_{m}$ represents the maximum sustained wind speed of a hurricane(in nautical miles per hour); $d_{i j}$ represents the distance between the hurricane eye and the distribution line $(i, j) ; R_{m w}$ represents the radius to the maximum wind speed (in nautical miles); $R_{s}$ indicates the radius of the area affected by the hurricane (in nautical miles); $\beta_{m w}$ indicates the modeling factor specifying the hurricane boundary, which is assumed to be 10 . The time-varying hurricane parameters, such as $v_{m}, R_{m w}, R_{s}$ and hurricane eye location can be simulated by the approach in [21].

3) Uncertainty of line repair time: The repair time of each damaged line, $T_{i j}^{R}(s)$, is a random variable that depends on utilities' dispatches of repair crews and resources [22], [23]. Without loss of generality, it is assumed that the repair time of each damaged line is independent and shares the same Weibull probability density function, $\forall(i, j) \in \Omega_{B}$ :

$$
f_{T_{i j}^{R}}(t)= \begin{cases}\frac{\beta_{T}}{\alpha_{T}}\left(\frac{t}{\alpha_{T}}\right)^{\beta_{T}-1} \exp \left[-\left(\frac{t}{\alpha_{T}}\right)^{\beta_{T}}\right] & \text { if } t \geqslant 0 \\ 0 & \text { otherwise, }\end{cases}
$$

where $\beta_{T}=10$ and $\alpha_{T}=4[16]$.

4) Uncertainty of line damage status: $\boldsymbol{u}\left(s ; \boldsymbol{x}^{h}\right)$ represents the outcome of the random event (whether the line is damaged in the extreme weather event) parametrized by the decision $\boldsymbol{x}^{h} . \boldsymbol{x}^{h}$ acts as a parameter, reflecting the endogeneity of the line damage uncertainty: the damage probability of a line will decrease if the line is hardened. Here comes the challenge: we cannot generate the specific scenarios,particularly the samples of $\boldsymbol{u}\left(s ; \boldsymbol{x}^{h}\right)$, in advance of solving the optimization problem, because $\boldsymbol{x}^{h}$ is a decision variable that is unknown beforehand. A brutal-force solution for this problem is to generate a dictionary of the scenario set, $\left\{\mathcal{S}_{x^{h}}\right\}$, for all possible values of $x^{h}$ in advance, then use proper second-stage constraints to look up the specific $\mathcal{S}_{\boldsymbol{x}^{h}}$ corresponding to the current $\boldsymbol{x}^{h}$. However, that is computationally intractable since the number of possible values of $\boldsymbol{x}^{h}$ boosts exponentially with the size of the system. 
We propose a modeling strategy to solve the challenges. Based on the independence among different lines, first we have $u_{i j, t}\left(s ; \boldsymbol{x}^{h}\right)=u_{i j, t}\left(s ; x_{i j}^{h}\right), \forall(i, j) \in \Omega_{B}$. Then, we decouple each $u_{i j, t}\left(s ; x_{i j}^{h}\right)$ into two independent parameters $\zeta_{i j, t}^{1}(s)$ and $\zeta_{i j, t}^{0}(s)$ (see [11), where $s$ is put as superscript). $\zeta_{i j, t}^{1}(s)$ represents the damage status of line $(i, j)$ if the line is hardened, while $\zeta_{i j, t}^{0}(s)$ represents the damage status of line $(i, j)$ if the line is not hardened. Constraint 11 picks $\zeta_{i j, t}^{1}(s)$ or $\zeta_{i j, t}^{0}(s)$ to represent $u_{i j, t}\left(s ; x_{i j}^{h}\right)$ according to the value of $x_{i j}^{h}$ as shown in Fig. 3 Notice that the distributions of $\zeta_{i j, t}^{1}(s)$ and $\zeta_{i j, t}^{0}(s)$ are conditionally independent of $\boldsymbol{x}^{h}$, thus can be determined in advance.

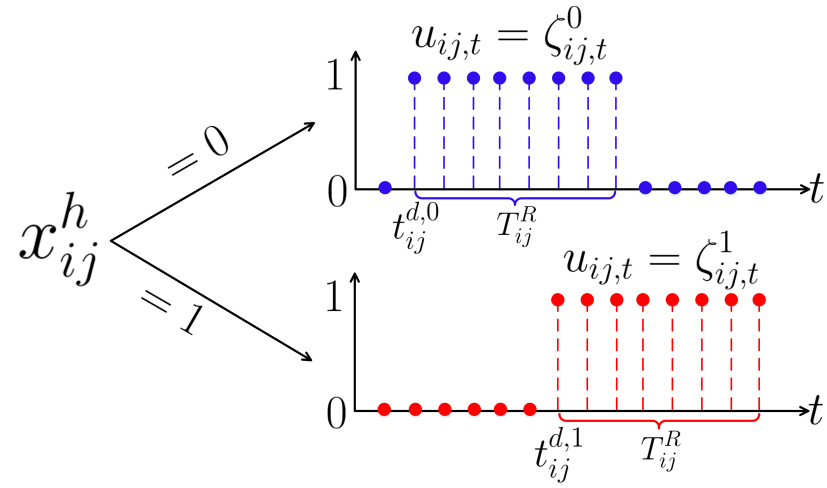

Fig. 3. The damage status of line $(i, j)$ with corresponding hardening decision

Therefore, we only need to sample $\zeta_{i j, t}^{1}(s)$ and $\zeta_{i j, t}^{0}(s)$ for all $(i, j)$ independently in the phase of scenario generation. For different $t$, the inter-temporal correlation imposed by the repair process is also considered in this paper. In particular, we have the following process to determine the damage status of a line during a hurricane. ( $i)$ Starting from $t=0, \zeta_{i j, t}^{0}(s)$, regarded as a function of $t$, is in a Bernoulli process with damage (failure) probability $p_{i j}(v)$ (explained later) until it equals 1 for the first time at $t=t_{i j}^{d, 0}(s)$. So, $\zeta_{i j, t}^{0}(s)=0$ for all $t<t_{i j}^{d, 0}(s)$. (ii) During the next $T_{i j}^{R}(s)$ hours, $\zeta_{i j, t}^{0}(s)$ is set to be 1 , indicating the line outage during the repairing period. (iii) If the repair is accomplished before the end of the hurricane, we assume the line will not be damaged again in the remaining time of this hurricane. $t_{i j}^{d, 0}(s)$ can be sampled from the geometric distribution with parameter $p_{i j}(v) . \zeta_{i j, t}^{1}(s)$ can be described by the same process except the damage probability is reduced to $\frac{1}{10} p_{i j}(v)$ due to the hardening.

The failure probability of line $(i, j)$, denoted by $p_{i j}(v)$ can be derived by failure probabilities of all its major components (the poles in this paper) with the assumption of independence of lines' failures [24]:

$$
p_{i j}(v)=1-\prod_{k=1}^{m_{i j}}\left(1-p_{I_{k}}(v)\right), \forall(i, j) \in \Omega_{B}
$$

where $m_{i j}$ is the total number of poles of line $(i, j)$, and $p_{I_{k}}(v)$ is the failure probability of the $k$ th pole. So a line fails if at least one pole fails. $p_{I_{k}}(v)$, regarded as a function of $v$, is a fragility curve for wind load obtained through fragility analyses, which usually satisfies the lognormal distribution of the wind speed $v$ [24]. In this paper, to simplify the problem, we assume that all poles at the same distribution line share the same fragility curve and the failure probabilities of all poles are independent for a fixed $v$.
In addition, constraint (12) indicates that each damaged line's repair cost is linearly proportional to the line's repair time. According to the report in [25], there are two factors that impact a line's repair cost: equipment replacement cost and crew hours. It is assumed that lines have the same equipment replacement cost denoted by $c^{R_{0}}$, but have different repair time, which is represented by the time summation of line damage statuses $u_{i j, t}^{s}$ with respect to time.

5) Scenario Generation: In summary, the procedure for scenario generation is as follows:

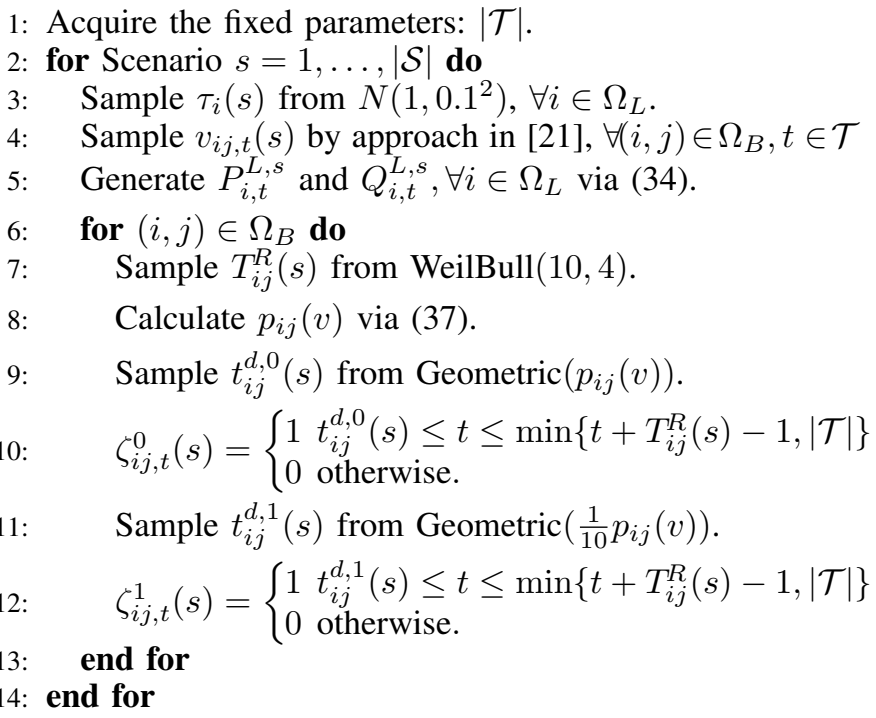

The procedure is independent of decision variables and can be run before solving the optimization problem (setting the parameters for the second-stage problems).

\section{Distribution System Modeling}

In this paper, the operation decisions of distribution systems are represented by recourse decisions to mitigate aftermath impacts of extreme weather events. These operation decisions consider network reconfiguration, load shedding, and DG redispatching. The operation constraints of distribution systems are represented by (13)-(32). Equations (13) and (14) represent the power balance at each bus. Equations (10), (15)-(19) give the full set of power flow constraints. They represent the generic power flow constraints for dynamic typologies. The switching actions are controlled by three binary variables: $x_{i j}^{c}$ indicates whether a tie switch is located in line $(i, j) ; u_{i j, t}^{s}$ indicates whether line $i j$ is damaged in the scenario $s$ at time $t$; and $y_{i j, t}^{c, s}$ indicates whether the tie switch, if exists, is on or off in the scenario $s$ at time $t$. The three variables for $(i, j)$ and $t$ determine whether the line is on or off at that time, whose logic is shown in Fig. 4.

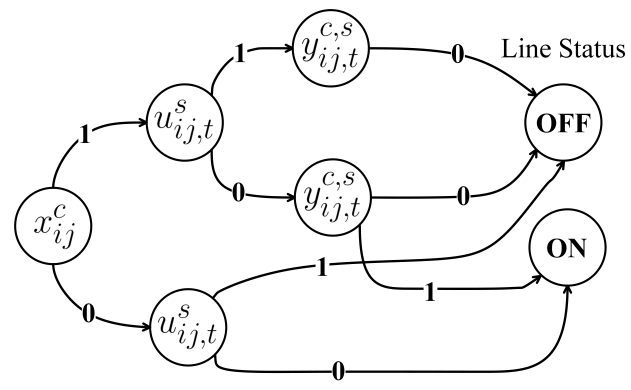

Fig. 4. The logic of line status 
Constraints (15)-(19) enforce the line flow limits, and represent the network connectivity in different typologies. In constraints (20)- 21 , the big $\mathrm{M}$ method is applied to ensure the voltages of two buses are independent if the line between them is damaged. In particular, when a line flow is forced to be zero, the voltages at the two ends will be decoupled. Distribution networks are normally operated as radial for effective coordination of protection systems under emergency and faulted operating conditions although they may have a meshed structure with the integration of DGs [26]. The radiality is enforced by constraints (22)-(26), which are based on the spanning tree approach in [10], [26]. Two binary variables $\vartheta_{m n, t}^{s}$ and $\vartheta_{n m, t}^{s}$ are introduced to define the spanning tree structure of distribution networks regardless of the power flow direction. Constraints $(22)$ and $(23)$ represent the relation between the connectivity status of line $(i, j)$ and the spanning tree variables $\vartheta_{m n, t}^{s}$ and $\vartheta_{n m, t}^{s}$. Constraint 22 indicates that line $(i, j)$ is in the spanning tree $\left(u_{i j, t}^{s}=0\right)$ if either node $m$ is the parent of node $n\left(\vartheta_{m n, t}^{s}=1\right)$, or node $n$ is the parent of node $m\left(\vartheta_{n m, t}^{s}=1\right)$. Constraints (23) indicates that if line $(i, j)$ is connected by closing the line switch, either $\vartheta_{m n, t}^{s}$ or $\vartheta_{n m, t}^{s}$ must be one. Equation (24) indicates that the substation node has no parent node, while constraint (25) requires that other nodes should have at most one parent node. Constraints (13)-(14) and (20)-(21) are linearized DistFlow equations which have been widely used to calculate the complex power flow and voltage profile in problems such as DG placement, service restoration, system operation, and planning of distribution systems [10], [13], [14], [18], [27]-[31]. Constraints (27] and (28) limit active and reactive power output of DGs, respectively. Constraint (31) imposes the voltage limits. Constraint (32) limits load shedding ratios. The load shedding is modeled as a continuous variable to reflect the evolving severity of extreme events and system damage states.

\section{Solution Algorithm}

In this section, we introduce two modified $\mathrm{PH}$ algorithms with and without bundling scenarios to solve the ROD problem presented in Section III Firstly, we use a compact notation to express the SMIP ROD model. In order to illustrate how the scenario-based PH works, we rewrite the compact notation of the SMIP ROD model into a scenario formulation. Secondly, the motivation and implementation steps of the modified $\mathrm{PH}$ algorithm for solving the SMIP ROD model are detailed. Thirdly, an approach to compute the lower bounds is presented to evaluate the solution quality of the modified PH algorithms. Fourthly, in order to accelerate the convergence of the original $\mathrm{PH}$ algorithm and improve its lower bound, we decompose the subproblems by scenario bundles instead of individual scenarios in the original $\mathrm{PH}$ algorithm.

\section{A. The Compact Notation of ROD Problem}

In order to elaborate the proposed algorithms and facilitate the solution discussion, we use a compact notation to express the proposed ROD model:

$$
\begin{aligned}
\min _{\boldsymbol{x}} \boldsymbol{c}^{\top} \boldsymbol{x} & +\sum_{s \in \mathcal{S}} p_{r}(s) \phi(\boldsymbol{x}, s) \\
\text { s.t. } & \boldsymbol{A} \boldsymbol{x} \leqslant \boldsymbol{b} \\
\boldsymbol{x} & \in \mathbb{Z}_{+}^{n_{1}}
\end{aligned}
$$

where vector $\boldsymbol{x}$ represents the binary decision variables in the first stage as shown in (4), and the cost coefficient vectors are defined as $c \in \mathbb{R}^{n_{1}}$. Constraint (39) is a vector form representation of the number limit of DGs and line switches in (2)-(3). $\phi(\boldsymbol{x}, s)$ denotes the system operation problem for a given scenario $s \in \mathcal{S}$, which is defined as follows:

$$
\begin{gathered}
\phi(\boldsymbol{x}, s)=\min \quad \boldsymbol{g}^{\top} \boldsymbol{y} \\
\text { s.t. } \quad \boldsymbol{F} \boldsymbol{y} \leqslant \boldsymbol{r}(s)-\boldsymbol{e}(s) \boldsymbol{x} \\
\boldsymbol{y} \in \mathbb{Z}_{+}^{p_{2}} \times \mathbb{R}^{n_{2}-p_{2}}
\end{gathered}
$$

where the vector $\boldsymbol{y}$ represents the second-stage decision variables. Constraints (10)- (32) can be expressed in a vector form as shown in (42). Here, $c \in \mathbb{R}^{n_{1}}, A \in \mathbb{R}^{m_{1} \times n_{1}}, b \in \mathbb{R}^{m_{1}}, g \in$ $\mathbb{R}^{n_{2}}, \boldsymbol{F} \in \mathbb{R}^{m_{2} \times n_{2}}, \boldsymbol{e} \in \mathbb{R}^{m_{2} \times n_{1}}, \boldsymbol{r} \in \mathbb{R}^{m_{2}}$ comprise the data of the SMIP ROD model.

The SMIP ROD model in the form of (38)-(43) is an optimization problem with an infinite dimension. In order to cope with this difficulty, we assume that there is a finite number of scenarios $s=1, \ldots,|\mathcal{S}|$, with the corresponding probabilities $p_{r}(s)=\frac{1}{|\mathcal{S}|}$. Then, the problem (38)-(43) can be written as a large-scale deterministic mixed-integer linear program (MILP) with a block-angular structure [32]:

$$
\begin{gathered}
z=\min \left\{\boldsymbol{c}^{\top} \boldsymbol{x}+\sum_{s=1}^{|\mathcal{S}|} p_{r}(s) \boldsymbol{g}(s)^{\top} \boldsymbol{y}(s):(\boldsymbol{x}, \boldsymbol{y}(s)) \in K(s),\right. \\
s=1, \ldots,|\mathcal{S}|\}
\end{gathered}
$$

where $K(s)=\left\{(\boldsymbol{x}, \boldsymbol{y}(s)): \boldsymbol{A} \boldsymbol{x} \leqslant \boldsymbol{b}, \boldsymbol{x} \in \mathbb{Z}_{+}^{n_{1}}, \boldsymbol{F} \boldsymbol{y}(s) \leqslant\right.$ $\left.\boldsymbol{r}(s)-\boldsymbol{e}(s) \boldsymbol{x}, \boldsymbol{y}(s) \in \mathbb{Z}_{+}^{p_{2}} \times \mathbb{R}^{n_{2}-p_{2}}\right\}$. Here the first stage decision vector $\boldsymbol{x}$ must be scenario independent.

By introducing copies of the first-stage variables $x$, the block-angular structure of (44) leads to the so-called scenario formulation of the SMIP ROD model:

$$
\begin{gathered}
z=\min \left\{\sum_{s=1}^{|\mathcal{S}|} p_{r}(s)\left(\boldsymbol{c}^{\top} \boldsymbol{x}(s)+\boldsymbol{g}(s)^{\top} \boldsymbol{y}(s)\right):(\boldsymbol{x}(s), \boldsymbol{y}(s))\right. \\
\in K(s), s=1, \ldots,|\mathcal{S}|, \boldsymbol{x}(1)=\cdots=\boldsymbol{x}(|\mathcal{S}|)\}
\end{gathered}
$$

where $\boldsymbol{x}(1)=\cdots=\boldsymbol{x}(|\mathcal{S}|)$ represents the non-anticipativity constraint, which will stipulate the first-stage decision vector $\boldsymbol{x}$ to be independent of scenarios. This scenario formulation (45) slips the large-scale SMIP ROD model into scenario subproblems with the condition of non-anticipativity constraints.

\section{B. Progressive Hedging Algorithm}

The general combination, NP-hard natural of mixed-integer problems and uncertainty, leads to the considerable difficulty in solving SMIP ROD models. For small-scale SMIP ROD problems, standard MILP solvers can be used to directly solve their extensive forms (EFs) presented in (44). However, for large-scale ROD problems, their EFs are too large to solve using in a reasonable run-time using available MIP solvers. In addition, the commonly used stage-based decomposition algorithm, Benders decomposition, relies heavily on the convexity of $\phi(\boldsymbol{x}, s)$ and cannot be directly applied to the case 
with integer variables in the second stage [33]. Rockafellar and Wets proposed the Progressive Hedging $(\mathrm{PH})$ decomposition algorithm as a heuristic [34] to effectively solve stochastic mixed-integer problems. $\mathrm{PH}$ algorithm can reduce the computational difficulty by decomposing the EF in (44) into scenariobased subproblems as shown in (45) and solving subproblems in parallel. PH has been successfully implemented in solving unit commitment problems [33], [35], [36].

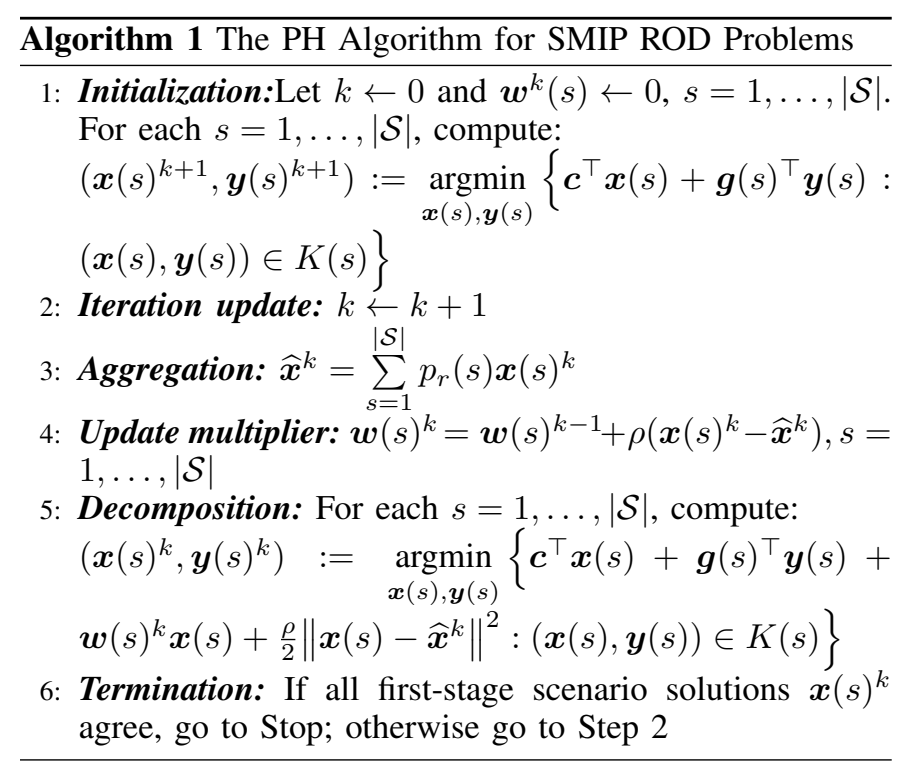

For such a two-stage SMIP ROD problem, the modified PH algorithm is sketched in Algorithm 1. The PH algorithm is initialized by solving the individual-scenario problems in Step 1. In each iteration, $\mathrm{PH}$ solves subproblems individually and aggregates the solutions to obtain the expected value $\widehat{\boldsymbol{x}}^{k}$. The estimates of multipliers $\boldsymbol{w}(s)^{k}$ are updated in Step 4 using a specific penalty parameter $\rho$ to enforce the non-anticipative policy. The performance of PH depends on the value of $\rho$. The decomposition step (Step 5) of each iteration involves solving variants of subproblems that are augmented with a linear term proportional to the multiplier $\boldsymbol{w}(s)^{k}$ and a squared two norm term penalizing deviation of $\boldsymbol{x}(s)^{k}$ from $\widehat{\boldsymbol{x}}^{k-1}$.

\section{Computation of lower bounds for $\mathrm{PH}$}

Computational studies have shown that $\mathrm{PH}$ can find solutions with acceptable optimality gap for practical applications [36]. However, it is still necessary to evaluate the quality of PH solutions. Gade et al. [37] proposed an approach to calculate the lower bounds in any iteration of $\mathrm{PH}$ by solving a simple optimization problem. We modify the proposition of lower bound in [37] for our proposed formulation:

Proposition 1: Let $\boldsymbol{w}(s)$, satisfies $\sum_{s=1}^{|\mathcal{S}|} p_{r}(s) \boldsymbol{w}(s)=0$. And $D_{s}(\boldsymbol{w}(s)):=\min \left\{p_{r}(s)\left(\boldsymbol{c}^{\top} \boldsymbol{x}(s)+\boldsymbol{g}(s)^{\top} \boldsymbol{y}(s)+\right.\right.$ $\boldsymbol{w}(s) \boldsymbol{x}(s)):(\boldsymbol{x}(s), \boldsymbol{y}(s)) \in K(s)\}$

Then the lower bound can be expressed as $D(\boldsymbol{w}):=$ $\sum_{s=1}^{|\mathcal{S}|} D_{s}(\boldsymbol{w}(s)) \leqslant z^{*}$.

In each iteration, the lower bound $D(\boldsymbol{w})$ on the optimal objective value $z^{*}$ is computed using the multiplier $\boldsymbol{w}(s)^{k}$.
These lower bounds are reported in our numerical results. The major advantage is that we can obtain the lower bounds for a stochastic mixed-integer problem even when the sub-problems are not solved to the optimality, and a lower bound can be easily calculated with approximately the same effort as one $\mathrm{PH}$ iteration.

\section{The PH Algorithm with Scenario Bundling}

Motivated by Crainic's strategy of grouping scenarios in PH-based meta-heuristics [38], Gade et al. [37] proposed the bundle version of $\mathrm{PH}$ algorithm, which allows Step 1 and Step 5 of PH algorithm to solve small EFs of the SMIP ROD model rather than single-scenario problems. The advantages over the basic PH are as follows: 1) scenario bundling can significantly improve the quality of PH algorithm's lower bounds [37]; 2) scenario bundling can accelerate the speed of agreement in the first-stage solutions, which in turn reduces the number of $\mathrm{PH}$ iterations for convergence [35]. However, the increased number of scenarios in each bundle may result in the increased computational difficulty of the bundles as each of them yields a small-scale extensive form stochastic program.

Suppose the set of scenarios is partitioned into bundles, each has $N_{S}$ scenarios. We denote the set of bundles by $\mathcal{B}$, with $\beta \in \mathcal{B}$ and $\mathcal{S}(\beta) \subset \mathcal{S}$ represents the scenarios in bundle $\beta$. So $|\mathcal{B}|=\frac{|\mathcal{S}|}{N_{S}}$. Let $p_{r}(\beta)=\sum_{s \in \mathcal{S}(\beta)} p_{r}(s), \beta \in \mathcal{B}$. Then the $\mathrm{PH}$ algorithm with scenario bundling is described in Algorithm 2. We restate the extension of Proposition 1 to the bundle version of $\mathrm{PH}$ algorithm here as Proposition 2.

Proposition 2: Let $\boldsymbol{w}(\beta), \beta \in \mathcal{B}$, satisfies $\sum_{\beta \in \mathcal{B}} p_{r}(\beta) \boldsymbol{w}(\beta)=$ 0 . Let

$$
\begin{gathered}
D_{\beta}(\boldsymbol{w}(\beta)):=\min \left\{p _ { r } ( \beta ) \left(\boldsymbol{c}^{\top} \boldsymbol{x}(\beta)+\sum_{s \in \mathcal{S}(\beta)} \frac{p_{r}(s)}{p_{r}(\beta)} \boldsymbol{g}(s)^{\top} \boldsymbol{y}(s)\right.\right. \\
\left.+\boldsymbol{w}(\beta) \boldsymbol{x}(\beta)):\left(\boldsymbol{x}(\beta),(\boldsymbol{y}(s))_{s \in \mathcal{S}(\beta)}\right) \in K(\beta)\right\}
\end{gathered}
$$$$
\text { Then } D(\boldsymbol{w}):=\sum_{\beta \in \mathcal{B}} D_{\beta}(\boldsymbol{w}(\beta)) \leqslant z^{*} \text {. }
$$

\section{NumericAl RESUlts}

Wind-induced extreme weather events (e.g. hurricane, tornado, ice storms) pose the top threat to overhead distribution systems. In this paper, we projected two test systems, 34- and 123-bus distribution feeders into two coastal cities in Texas where hurricanes occur frequently. According to the histogram of landfall hurricane frequency in Texas [39], we assume $\omega_{H}=2$. The detailed network and load data can be found in [40], [41] and [42]. It is assumed that a city close to Texas coast will be affected by a hurricane for 24 hours.

The initial investment costs of the three considered ROD measures are shown in Table [II It is assumed the life time of the three ROD measures is 10 years. Without considering the interest rate, the annual capital cost for purchasing and installation of each ROD measure is $\frac{1}{10}$ of the initial investment cost. Here we use the natural gas-fired CHPs with $400 \mathrm{~kW}$ capacity as back-up DGs, which can be controlled by utilities for enhancing network resilience. The candidate positions to implement different ROD measures are shown in Table I. In practice, the candidate positions to install tie switches are usually restricted by many considerations such as the necessity 
Algorithm 2 The PH Algorithm for ROD Problems with Scenario Bundling

1: Initialization:Let $k \leftarrow 0$ and $\boldsymbol{w}(\beta)^{k} \leftarrow 0$. For each $\beta \in \mathcal{B}$, compute:

$$
\begin{gathered}
\left(\boldsymbol{x}(\beta)^{k+1},\left(\boldsymbol{y}(s)^{k+1}\right)_{s \in \mathcal{S}(\beta)}\right):=\underset{\boldsymbol{x}(\beta), \boldsymbol{y}(s)}{\operatorname{argmin}}\left\{\boldsymbol{c}^{\top} \boldsymbol{x}(\beta)+\right. \\
\left.\sum_{s \in \mathcal{S}(\beta)} \frac{p_{r}(s)}{p_{r}(\beta)} \boldsymbol{g}(s)^{\top} \boldsymbol{y}(s):\left(\boldsymbol{x}(\beta),(\boldsymbol{y}(s))_{s \in \mathcal{S}(\beta)}\right) \in K(\beta)\right\}
\end{gathered}
$$

2: Iteration update: $k \leftarrow k+1$

3: Aggregation: $\widehat{\boldsymbol{x}}^{k}:=\sum_{\beta \in \mathcal{B}} p_{r}(\beta) \boldsymbol{x}(\beta)^{k}$

4: Update multiplier: $\boldsymbol{w}(\beta)^{k}=\boldsymbol{w}(\beta)^{k-1}+\rho\left(\boldsymbol{x}(\beta)^{k}-\widehat{\boldsymbol{x}}^{k}\right)$, $\beta \in \mathcal{B}$

5: Decomposition: For each $\beta \in \mathcal{B}$, compute:

$$
\begin{aligned}
& \left(\boldsymbol{x}(\beta)^{k+1},\left(\boldsymbol{y}(s)^{k+1}\right)_{s \in \mathcal{S}(\beta)}\right):=\underset{\boldsymbol{x}(\beta), \boldsymbol{y}(s)}{\operatorname{argmin}}\left\{\boldsymbol{c}^{\top} \boldsymbol{x}(\beta)+\right. \\
& \sum_{s \in \mathcal{S}(\beta)} \frac{p_{r}(s)}{p_{r}(\beta)} \boldsymbol{g}(s)^{\top} \boldsymbol{y}(s)+\underset{\boldsymbol{w}(\beta)^{k-1} \boldsymbol{x}(\beta)}{+}+ \\
& \left.\frac{\rho}{2}\left\|\boldsymbol{x}(\beta)-\widehat{\boldsymbol{x}}^{k-1}\right\|^{2}:\left(\boldsymbol{x}(\beta),(\boldsymbol{y}(s))_{s \in \mathcal{S}(\beta)}\right) \in K(\beta)\right\}
\end{aligned}
$$

6: Termination: If all first-stage scenario solutions $\boldsymbol{x}_{\beta}^{k}$ agree, go to Stop; otherwise go to Step 2

and space. Hence, the candidate positions for switches are predefined based on the optimal switch allocations for network reconfiguration in $[43]-[45]$. The candidate switch locations of 34-Bus and 123-Bus test systems are shown in Fig. 5 and Fig. 6 respectively.

TABLE I

The Feasible Regions FOR THE Three ROD MEAsures

\begin{tabular}{ccccc}
\hline \multirow{2}{*}{ No. } & ROD Measure & Candidate positions & \multicolumn{2}{c}{ Number of Variables } \\
& & & 34-Bus & 123-Bus \\
\hline 1 & Back-up DG deployment & All nodes & 34 & 123 \\
2 & Line hardening & All line sections & 37 & 128 \\
3 & Tie switch deployment & Pre-selected lines & 9 & 14 \\
\hline
\end{tabular}

The basic load shedding penalty cost is assumed to be $\$ 14 / k W h$ [46] and the load shedding cost parameter $c_{i}^{L}$ in equation (9] is the product of the basic load shedding penalty cost and the load priority. It is assumed that there are 5 load priorities and the voltage range is set as 0.95p.u $\sim 1.05 p . u$. The time-period length is assumed to be $\Delta t=1$ hour. The initial repair cost $c^{R_{0}}$ of each line is assumed as $\$ 2000$.

TABLE II

The Capital Cost of Different ROD Measures

\begin{tabular}{ccc}
\hline \#No. & Measures & Cost $(\$)$ \\
\hline 1 & Hardening line & $5,924 /$ pole $[47]$ \\
2 & Installing a back-up DG & $1,500 / k W[15]$ \\
3 & Adding an automatic tie switch & $15,000[15]$ \\
\hline *Assume the span of two consecutive poles is $150 \mathrm{ft}$.
\end{tabular}

\section{A. Computational platforms}

All models and algorithms are implemented using the PySP package in Pyomo. All of our customizations relating to $\rho$ setting, variable fixing, cycle detection/breaking, and variable slamming are performed through a parametrization of PySP's WW extension module. IBM's CPLEX 12.6 mixed-integer solver is used to solve all subproblems. All experiments are implemented on Iowa State University Condo cluster, whose individual blades consist of two 2.6 GHz 8-Core Intel E5-2640 v3 processors and 128GB of RAM.

\section{B. Experiment Results}

The parallel PH algorithm implementation is performed on a modest-sized 34-bus distribution system with 50 scenarios and a large-scale 123-bus distribution system with 100 scenarios. The scenarios are randomly generated via procedures described in Section II-B5. We consider both run-time and overall solution quality in the analysis. The $\mathrm{PH}$ algorithms are performed in parallel, utilizing 4 nodes of the Condo cluster for 34-bus system and 7 nodes for 123-bus system. We also solve the EF of ROD problems on both test systems by allocating the maximum possible number of threads (16) to each CPLEX running on a node. The solution of EF, which provides the global optimal solution and an indication of absolute instance difficulty [48], serves as a performance baseline for $\mathrm{PH}$ algorithms.

Comparison of $\mathrm{PH}$ algorithm with and without bundling scenarios: We set the optimality tolerance of $\mathrm{PH}$ for solving subproblems as $10^{-5}$. The $\mathrm{PH}$ convergence metric is assumed as $0.0001 \%$, which is defined as the unscaled sum of difference between the first-stage variables and the means.

For the 34-bus ROD problem, the EF of this 50-scenario instance has $1,608,766$ variables $(1,361,866$ binary) and 712,050 constraints. We solve the EF in 6.5733 hours of wall clock time with a $0.01 \%$ optimality gap. The first-stage investment cost of the EF is $\$ 3,987,000$. Then we experimented $\mathrm{PH}$ with different numbers of scenarios per bundle. For example, 10 bundles means the 50 scenarios are divided into 10 bundles with 5 scenarios in each. The results are shown in Tables III The first column in Table III indicates the number of bundles while the second column represents the total cost of the first stage and the second stage. The third column in this table describes the lower bound of $\mathrm{PH}$, while the fourth column represents the relative gap between the objective and lower bound. The lower bound is computed at the last iteration. The fifth column describes the first-stage cost, while the sixth and seventh columns respectively report the convergence iteration and run time. The optimal ROD investment decisions are shown in Figs. 5. By bundling scenarios into 10 bundles, the lower bound of $\mathrm{PH}$ algorithm is much tighter with a $2.04 \%$ relative gap, and the algorithm converges with less iterations. However, it takes longer time to solve sub-problems.

For the large-scale 123-bus ROD problem, the EF of the 100 -scenario instance has $39,012,195$ variables $(37,282,600$ binary) and 4,659,201 constraints. No feasible incumbent solution of the EF is obtained by the end of 240-hour wall time run, which shows the significant computational challenge of large-scale stochastic ROD problems when being tackled by non-decomposition-based methods. On the other hand, we show the results of $\mathrm{PH}$ algorithms with bundling in Table IV Although the lower bounds are not as tight as those in Table III, they are within an acceptable range, compared with results obtained by a linear relaxation method in [36] with a lower bound of $227,710.98$. Overall, we obtain the best tradeoff between computational burdens and solution quality using bundles with 2 scenarios as shown in Fig. 6 These 
results demonstrate the effectiveness of bundling as a method for improving lower bound quality and convergence speed.

TABLE III

THE COMPARISON OF PH ALGORITHM WITH AND Without BUNDLING SCENARIOS IN 34-BUS DISTRIBUTION SYSTEM

\begin{tabular}{ccccccc}
\hline Bundles & Obj. & PH L.B. & $\begin{array}{c}\text { Relative } \\
\text { Gap }\end{array}$ & $\begin{array}{c}\text { First Stage } \\
\text { Cost }\end{array}$ & Iter. & $\begin{array}{c}\text { Run } \\
\text { Time }\end{array}$ \\
\hline No & $718,827.69$ & $681,163.90$ & $5.24 \%$ & 398,700 & 168 & $0.65 \mathrm{hr}$ \\
25 & $718,827.69$ & $6,87,746.06$ & $4.32 \%$ & 398,700 & 68 & $0.24 \mathrm{hr}$ \\
10 & $718,827.69$ & $704,157.59$ & $2.04 \%$ & 398,700 & 28 & $1.78 \mathrm{hr}$ \\
\hline
\end{tabular}

TABLE IV

THE COMPARISON OF PH ALgORITHM WITH AND WiTHOUT BUNDLING SCENARIOS IN THE MODIFIED 123-Bus FEEDERS

\begin{tabular}{ccccccc}
\hline \multirow{2}{*}{ Bundles } & \multirow{2}{*}{ Obj. } & PH L.B. & $\begin{array}{c}\text { Relative } \\
\text { Gap }\end{array}$ & $\begin{array}{c}\text { First Stage } \\
\text { Cost }\end{array}$ & Iter. & $\begin{array}{c}\text { Run } \\
\text { Time }\end{array}$ \\
\hline No & $1,874,209.49$ & $1,634,251.87$ & $12.65 \%$ & 258,600 & 300 & $35.74 \mathrm{hr}$ \\
50 & $1,867,431.12$ & $1,790,492.96$ & $4.12 \%$ & 258,600 & 167 & $42.73 \mathrm{hr}$ \\
25 & $1,870,939.95$ & $1,714,716.46$ & $8.35 \%$ & 261,000 & 95 & $54.84 \mathrm{hr}$ \\
\hline
\end{tabular}

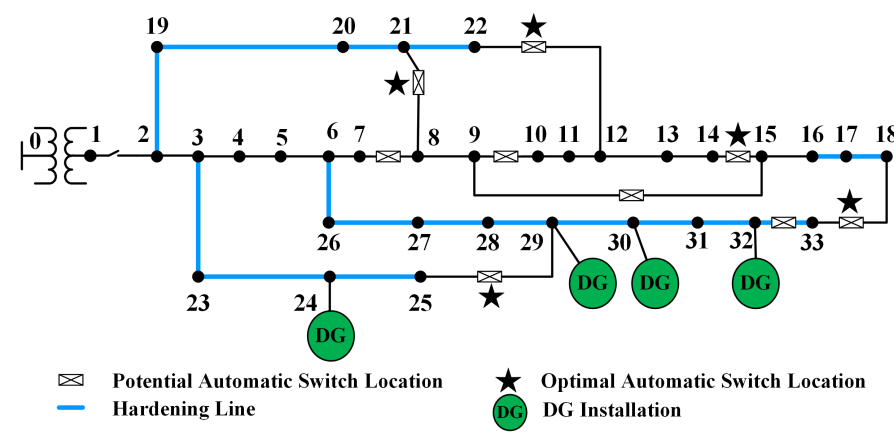

Fig. 5. The optimal ROD investment decisions of PH algorithm in 34-bus feeder

Solution validation by multiple replication procedure: In this paper, the Monte-Carlo simulation is used to generates 50 scenarios for 34-bus distribution system. However, the complete scenario space is extremely large. Hence it is necessary to test the quality of the solution based on limited generated damage scenarios. For this purpose, MRP [49] is used to test the stability and quality of the candidate solution shown in Fig. 5. The candidate solution is tested against 30 samples, each with a sample size of 50 scenarios. The one-sided confidence interval (CI) in the percentage term with regard to objective value for the optimality gap is $[0,0.64 \%]$ with $\alpha=0.05$, which means that there is a chance of $95 \%$ that the optimality gap is within the CI. We also do the same MPR for 123-bus distribution system, and its one-sided CI in the percentage term with regard to objective value for the optimality gap is $[0,7.52 \%]$ with $\alpha=0.05$. Thus, the candidate solutions of the stochastic programming for both test systems are stable and of high quality.

\section{CONClusion}

This paper proposes an innovative two-stage stochastic mixed-integer model to design resilient distribution systems against extreme weather events. The first stage is to make ROD decisions, which includes hardening existing distribution lines or deploying ROD resources such as back-up DGs and automatic tie switches. The second stage is to evaluate the

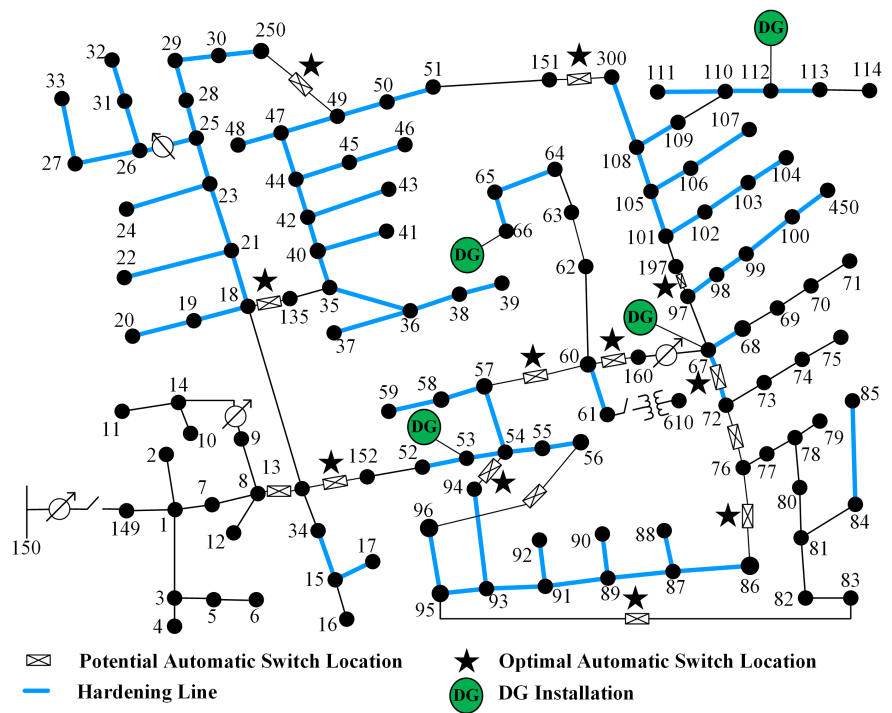

Fig. 6. The optimal ROD investment decisions of PH algorithm with 50 bundles in modified 123-bus feeder

system operation cost during extreme weather event. Four random variables are considered: load level multiplier, wind speed of extreme events, line repair time, and line damage status. As the first-stage hardening decisions affect the uncertainty realization of line damage statuses, a novel modeling strategy is presented to decouple the interdependency between line damage uncertainty and first-stage hardening decisions. The implementation of multiple ROD measures results in the non-convexity of the second-stage value function under integer recourses and considerable computational difficulties. The proposed PH algorithms with and without bundling scenarios, overcome these fundamental difficulties by decomposing the EF into scenario-based subproblems and solving these subproblems in parallel. Case studies on 34-bus and 123-bus distribution systems illustrate the efficiency and effectiveness of the proposed models and algorithms. The results also validate that the $\mathrm{PH}$ algorithm with scenario bundling can improve the overall solution quality.

\section{REFERENCES}

[1] R. E. Brown, "Hurricane hardening efforts in Florida," in Proc. IEEE Power and Energy Society General Meeting - Conversion and Delivery of Electrical Energy in the 21st Century, Jul. 2008, pp. 1-7.

[2] NERC, "Hurricane sandy event analysis report," Jan. 2014.

[3] ASCE, "ASCE report card on America's civil infrastructure systems," American Society of Civil Engineers, Reston, VA, Tech. Rep., 2013.

[4] A. Gholami, F. Aminifar, and M. Shahidehpour, "Front lines against the darkness: enhancing the resilience of the electricity grid through microgrid facilities," IEEE Electrif. Mag., vol. 4, no. 1, pp. 18-24, 2016.

[5] M. Panteli and P. Mancarella, "The grid: Stronger, bigger, smarter?: Presenting a conceptual framework of power system resilience," IEEE Power Energy Mag., vol. 13, no. 3, pp. 58-66, May 2015.

[6] H. Gao, Y. Chen, S. Mei, S. Huang, and Y. Xu, "Resilience-oriented prehurricane resource allocation in distribution systems considering electric buses," Proc. IEEE, 2017.

[7] H. Gao, Y. Chen, Y. Xu, and C.-C. Liu, "Resilience-oriented critical load restoration using microgrids in distribution systems," IEEE Trans. Smart Grid, vol. 7, no. 6, pp. 2837-2848, 2016.

[8] Y. Xu, C.-C. Liu, K. Schneider, F. Tuffner, and D. Ton, "Microgrids for service restoration to critical load in a resilient distribution system," IEEE Trans. Smart Grid, 2016.

[9] A. Arif and Z. Wang, "Service restoration in resilient power distribution systems with networked microgrid," in Proc. IEEE Power and Energy Society General Meeting (PESGM), Jul. 2016, pp. 1-5.

[10] A. Arif, Z. Wang, J. Wang, and C. Chen, "Power distribution system outage management with co-optimization of repairs, reconfiguration, and dg dispatch," IEEE Trans. Smart Grid, vol. PP, no. 99, p. 1, 2017. 
[11] C. Wang, Y. Hou, F. Qiu, S. Lei, and K. Liu, "Resilience enhancement with sequentially proactive operation strategies," IEEE Trans. Power Syst., vol. 32, no. 4, pp. 2847-2857, Jul. 2017.

[12] Y. Xu, C.-C. Liu, K. P. Schneider, and D. T. Ton, "Placement of remote-controlled switches to enhance distribution system restoration capability," IEEE Trans. Power Syst., vol. 31, no. 2, pp. 1139-1150, 2016.

[13] W. Yuan, J. Wang, F. Qiu, C. Chen, C. Kang, and B. Zeng, "Robust optimization-based resilient distribution network planning against natural disasters," IEEE Trans. Smart Grid, vol. PP, no. 99, pp. 1-10, 2016.

[14] S. Ma, B. Chen, and Z. Wang, "Resilience enhancement strategy for distribution systems under extreme weather events," IEEE Trans. Smart Grid, vol. PP, no. 99, p. 1, 2016.

[15] E. Yamangil, R. Bent, and S. Backhaus, "Resilient upgrade of electrical distribution grids." in AAAI, 2015, pp. 1233-1240.

[16] A. Arab, A. Khodaei, S. K. Khator, K. Ding, V. A. Emesih, and Z. Han, "Stochastic pre-hurricane restoration planning for electric power systems infrastructure," IEEE Trans. Smart Grid, vol. 6, no. 2, pp. 1046-1054, Mar. 2015.

[17] Z. Wang and J. Wang, "Self-healing resilient distribution systems based on sectionalization into microgrids," IEEE Trans. Power Syst., vol. 30, no. 6, pp. 3139-3149, Nov. 2015

[18] Z. Wang, B. Chen, J. Wang, and C. Chen, "Networked microgrids for self-healing power systems," IEEE Trans. Smart Grid, vol. 7, no. 1, pp. 310-319, Jan. 2016

[19] Z. Wang, J. Wang, B. Chen, M. M. Begovic, and Y. He, "MPCbased voltage/var optimization for distribution circuits with distributed generators and exponential load models," IEEE Trans. Smart Grid, vol. 5, no. 5, pp. 2412-2420, Sep. 2014.

[20] X. Chen, W. Wu, B. Zhang, and X. Shi, "A robust approach for active distribution network restoration based on scenario techniques considering load and dg uncertainties," in Proc. IEEE Power and Energy Society General Meeting (PESGM), Jul. 2016, pp. 1-5.

[21] P. Javanbakht and S. Mohagheghi, "A risk-averse security-constrained optimal power flow for a power grid subject to hurricanes," Electric Power Systems Research, vol. 116, pp. 408-418, 2014.

[22] A. Arif, S. Ma, and Z. Wang, "Optimization of transmission system repair and restoration with crew routing," in Proc. North American Power Symp. (NAPS), Sep. 2016, pp. 1-6.

[23] A. Arif and Z. Wang, "Networked microgrids for service restoration in resilient distribution systems," Transmission Distribution IET Generation, vol. 11, no. 14, pp. 3612-3619, 2017.

[24] A. M. Salman, Y. Li, and M. G. Stewart, "Evaluating system reliability and targeted hardening strategies of power distribution systems subjected to hurricanes," Reliability Engineering \& System Safety, vol. 144, pp. 319-333, 2015.

[25] L. Xu and R. Brown, "Undergrounding assessment phase 3 report: Ex ante cost and benefit modeling," Quanta Technology, Tech. Rep., 2008.

[26] R. A. Jabr, R. Singh, and B. C. Pal, "Minimum loss network reconfiguration using mixed-integer convex programming," IEEE Trans. Power Syst., vol. 27, no. 2, pp. 1106-1115, May 2012.

[27] M. E. Baran and F. F. Wu, "Network reconfiguration in distribution systems for loss reduction and load balancing," IEEE Trans. Power Delivery, vol. 4, no. 2, pp. 1401-1407, Apr. 1989.

[28] Z. Wang, B. Chen, J. Wang, J. Kim, and M. M. Begovic, "Robust optimization based optimal dg placement in microgrids," IEEE Trans. Smart Grid, vol. 5, no. 5, pp. 2173-2182, Sep. 2014.

[29] C. Chen, J. Wang, F. Qiu, and D. Zhao, "Resilient distribution system by microgrids formation after natural disasters," IEEE Trans. Smart Grid, vol. 7, no. 2, pp. 958-966, Mar. 2016.

[30] Z. Wang, B. Chen, J. Wang, and J. kim, "Decentralized energy management system for networked microgrids in grid-connected and islanded modes," IEEE Trans. Smart Grid, vol. 7, no. 2, pp. 1097-1105, Mar. 2016.

[31] A. Arif, S. Ma, and Z. Wang, "Online decomposed optimal outage management after natural disasters," in Proc. IEEE Power Energy Society General Meeting, Jul. 2017, pp. 1-5.

[32] G. Guo, G. Hackebeil, S. M. Ryan, J.-P. Watson, and D. L. Woodruff, "Integration of progressive hedging and dual decomposition in stochastic integer programs," Operations Research Letters, vol. 43, no. 3, pp. 311316,2015

[33] W. Römisch and S. Vigerske, "Recent progress in two-stage mixedinteger stochastic programming with applications to power production planning," in Handbook of Power Systems I. Springer, 2010, pp. 177208.

[34] R. T. Rockafellar and R. J.-B. Wets, "Scenarios and policy aggregation in optimization under uncertainty," Math. Oper. Res., vol. 16, no. 1, pp. 119-147, 1991

[35] S. M. Ryan, R. J.-B. Wets, D. L. Woodruff, C. Silva-Monroy, and J.-P Watson, "Toward scalable, parallel progressive hedging for stochastic unit commitment," in 2013 IEEE Power \& Energy Society General Meeting. IEEE, 2013, pp. 1-5.
[36] F. D. Munoz and J.-P. Watson, "A scalable solution framework for stochastic transmission and generation planning problems," Computational Management Science, vol. 12, no. 4, pp. 491-518, 2015.

[37] D. Gade, G. Hackebeil, S. M. Ryan, J.-P. Watson, R. J.-B. Wets, and D. L. Woodruff, "Obtaining lower bounds from the progressive hedging algorithm for stochastic mixed-integer programs," Math. Program., vol. 157 , no. 1, pp. 47-67, 2016.

[38] T. G. Crainic, M. Hewitt, and W. Rei, "Scenario grouping in a progressive hedging-based meta-heuristic for stochastic network design," Computers \& Operations Research, vol. 43, pp. 90 - 99, 2014.

[39] R. Brown, "Cost-benefit analysis of the deployment of utility infrastructure upgrades and storm hardening programs," Quanta Technology, Raleigh, 2009.

[40] F. Capitanescu, I. Bilibin, and E. R. Ramos, "A comprehensive centralized approach for voltage constraints management in active distribution grid," IEEE Trans. Power Syst., vol. 29, no. 2, pp. 933-942, March 2014.

[41] J. Zhu, "Optimal reconfiguration of electrical distribution network using the refined genetic algorithm," Electr. Power Syst. Res., vol. 62, no. 1, pp. $37-42,2002$.

[42] W. H. Kersting, "Radial distribution test feeders," in Power Engineering Society Winter Meeting, 2001. IEEE, vol. 2. IEEE, 2001, pp. 908-912.

[43] M. Sedighizadeh, M. Dakhem, M. Sarvi, and H. H. Kordkheili, "Optimal reconfiguration and capacitor placement for power loss reduction of distribution system using improved binary particle swarm optimization," International Journal of Energy and Environmental Engineering, vol. 5, no. 1, p. 73, 2014.

[44] H. Jiang, F. Ding, Y. Zhang, H. Jiang, F. Ding, and Y. Zhang, "Short-term load forecasting based automatic distribution network reconfiguration: Preprint," National Renewable Energy Laboratory (NREL), Golden, CO (United States), Tech. Rep., 2017.

[45] S. Chouhan, H. Inan, and A. Feliachi, "Optimal number and placement of automated sectionalizing switches for smart grid distribution automation," in Power and Energy Society General Meeting (PESGM), 2016. IEEE, 2016, pp. 1-5.

[46] R. E. Brown, Electric power distribution reliability. CRC press, 2008.

[47] D. Louth, "Governor's two-storm panel: Distribution infrastructure hardening options and recommendations," Connecticut Light \& Power, Tech. Rep., 2011.

[48] K. Cheung, D. Gade, C. Silva-Monroy, S. M. Ryan, J.-P. Watson, R. J.-B. Wets, and D. L. Woodruff, "Toward scalable stochastic uni commitment," Energy Syst., vol. 6, no. 3, pp. 417-438, 2015.

[49] G. Bayraksan and D. P. Morton, "Assessing solution quality in stochastic programs via sampling," Tutorials in Operations Research, vol. 5, pp. $102-122,2009$

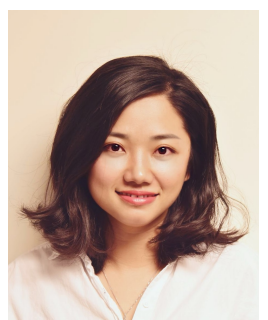

Shanshan Ma received the B.S. degree in information and electrical engineering from Zhejiang University City College, Hangzhou, China, in 2012, and the M.S. degree from the Department of Electrical Engineering and Computer Science, South Dakota State University, Brookings, SD, USA, in 2015. She is currently working toward a Ph.D. degree with the Department of Electrical \& Computer Engineering at Iowa State University, Ames, IA, USA Her current research interests include power distribution system resiliency, stochastic mixed-integer programming with applications in power systems, and electric power market design.

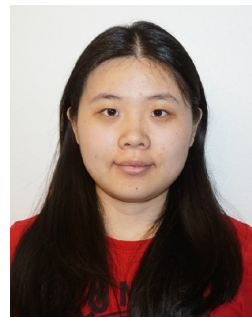

Liu Su received the B.S. degree in industrial engineering from Huazhong University of Science and Technology, Wuhan, China, in 2013 and her M.S. degree from the Department of Industrial and Manufacturing Systems Engineering, lowa State University, Ames, IA, in 2015. She is now pursing the Ph.D. degree in the Department of Industrial and Management Systems Engineering, University of South Florida and working as a research aide intern at Mathematics and Computer Science Division, Argonne National Laboratory, Argonne, IL, USA. Her research interests include optimization theories 


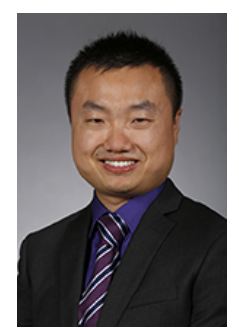

Zhaoyu Wang (S'13-M'15) is the Harpole-Pentair Assistant Professor with Iowa State University. $\mathrm{He}$ received the B.S. and M.S. degrees in electrical engineering from Shanghai Jiaotong University in 2009 and 2012, respectively, and the M.S. and Ph.D. degrees in electrical and computer engineering from Georgia Institute of Technology in 2012 and 2015, respectively. He was a Research Aid at Argonne National Laboratory in 2013, and an Electrical Engineer at Corning Inc. in 2014. His research interests include power distribution systems, microgrids, renewable integration, power system resiliency, demand response and voltage/VAR control. Dr. Wang received the IEEE PES General Meeting Best Paper Award in 2017 and the IEEE Industrial Application Society Prize Paper Award in 2016. Dr. Wang serves as the Secretary of IEEE PES Awards Subcommittee. He is an editor of IEEE TRANSACTIONS ON SMART GRID and IEEE POWER ENGINEERING LETTERS. His research projects are currently funded by the U.S. National Science Foundation, the U.S. Department of Energy, National Laboratories, PSERC, Iowa Economic Development Agency and industry.

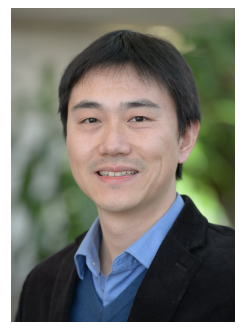

Feng Qiu (M'14) received his Ph.D. from the School of Industrial and Systems Engineering at the Georgia Institute of Technology in 2013. He is a computational scientist with the Energy Systems Division at Argonne National Laboratory, Argonne, IL, USA. His current research interests include optimization in power system operations, electricity markets, and power grid resilience.

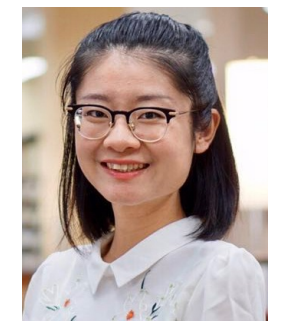

Ge Guo is a Ph.D. candidate in the Department of Industrial and Manufacturing System Engineering at Iowa State University, Ames Iowa. She received the B.S. degree from Beijing Institute of Graphic Communication, Beijing, China, in 2011, and the M.S. degree in Industrial Engineering from Texas A\&M University, College Station, Texas, in 2013.

Her research interests include optimization modeling and solution algorithm development on stochastic mixed-integer programming with applications in power systems, manufacturing systems and revenue management. 